\title{
Multi-Cuspon Solutions of the Wadati-Konno-Ichikawa Equation by Riemann-Hilbert Problem Method
}

\author{
Youzhi Tu \\ College of Sciences, University of Shanghai for Science and Technology, Shanghai, China \\ Email: tuyz2018@163.com
}

How to cite this paper: Tu, Y.Z. (2020) Multi-Cuspon Solutions of the Wadati-Konno-Ichikawa Equation by RiemannHilbert Problem Method. Open Journal of Applied Sciences, 10, 100-109. https://doi.org/10.4236/ojapps.2020.103008

Received: February 21, 2020

Accepted: March 16, 2020

Published: March 19, 2020

Copyright $\odot 2020$ by author(s) and Scientific Research Publishing Inc. This work is licensed under the Creative Commons Attribution International License (CC BY 4.0).

http://creativecommons.org/licenses/by/4.0/

\section{(c) (i) Open Access}

\begin{abstract}
In this paper, we consider the initial value problem for a complete integrable equation introduced by Wadati-Konno-Ichikawa (WKI). The solution $q(x, t)$ is reconstructed in terms of the solution of a $2 \times 2$ matrix Riemann-Hilbert problem via the asymptotic behavior of the spectral variable at one non-singularity point, i.e., $\lambda=0$. Then, the one-cuspon solution, two-cuspon solutions and three-cuspon solution are discussed in detail. Further, the numerical simulations are given to show the dynamic behaviors of these soliton solutions.
\end{abstract}

\section{Keywords}

WKI Equation, Initial Value Problem, Cuspon Solution, Riemann-Hilbert Problem

\section{Introduction}

The initial value problem for the nonlinear integrable equation

$$
\begin{aligned}
& q_{t}+\left[\frac{q_{x}}{\left(1+q^{2}\right)^{3 / 2}}\right]_{x x}=0, \quad t>0, \quad-\infty<x<+\infty, \\
& q(x, 0)=q_{0}(x), \quad-\infty<x<+\infty,
\end{aligned}
$$

where we assume $q_{0}(x)$ decays to 0 sufficiently fast, was derived Wadati, Konno and Ichikawa (WKI) in [1] [2]. This equation can be used to describe nonlinear transverse oscillations of elastic beams under tension [3] [4] and the motion of curves in $E^{3}$ [5]. If beam is flexible enough, it could be deformed into a shape of loop, of which upper half portion takes the negative curvature. One 
can easily realize such a situation on a stretched rope. Compared with the already known systems such as the nonlinear Schrödinger (NLS) equation and the derivative nonlinear Schrödinger (DNLS) equation, Equation (1) is highly nonlinear and even has the saturation effects [1]. Therefore its analysis is quite interesting mathematically and physically.

For the WKI Equation (1), many researchers have done a lot of studies and obtained many classical conclusions. There are so many interesting works on this equation in these years. Wadati, Konno and Ichikawa presented two types of nonlinear equations, and showed the equations have an infinite number of conservation laws and can be expressed in the Hamiltonian form in [1]. The authors of [6] studied the WKI equation by the inverse scattering transform (IST) method [7], obtained a one-soliton solution and a two-soliton solution, and analyzed some properties. In [8], the authors showed that two inverse scattering formalisms by Ablowitz, Kaup, Newell and Segur and by Wadati, Konno and Ichikawa are connected through a gauge transformation, and one-soliton solutions of equations associated with the W-K-I scheme are also examined. The authors derived the WKI equation from motion of curves in $E^{3}$ and gave the corresponding group-invariant solutions [5]. However, the three-soliton solution of the WKI equation hasn't been discussed via the Riemann-Hilbert problem and given relevant numerical simulations.

In this paper, the one-cuspon solution, two-cuspon solutions and three-cuspon solution of the WKI equation were discussed via the Riemann-Hilbert problem. And the numerical simulations were given to show the dynamic behaviors of these cuspon solutions. Besides, the novelty of this paper is twofold. First, the solution of the WKI Equation (1) is reconstructed via a $2 \times 2$ matrix Riemann-Hilbert problem at $\lambda \rightarrow 0$ instead of $\lambda \rightarrow \infty$, although $\lambda=0$ is not the singularity point of the Lax pair (2). Second, we obtain multi-cuspon solutions, which were not discussed before.

This paper is organized as follows. In Section 2, we perform the spectral analysis at $\lambda=\infty$ and $\lambda=0$, respectively. Then reconstruct the solution $q(x, t)$ in terms of the solution of the associated Riemann-Hilbert problem for the WKI equation in variable $(y, t)$ instead of $(x, t)$ via $\lambda \rightarrow 0$. In Section 3 , assuming $a(\lambda)$ only has simple zeros, we obtain the algebraic system of $\mathrm{N}$-soliton solutions. We give the details for $N=1,2,3$ and the numerical simulations of these cuspon solutions.

\section{Spectral Analysis and Riemann-Hilbert Problem}

\subsection{Lax Pair}

The Lax pair of the WKI equation is

$$
\psi_{x}=U \psi, \quad \psi_{t}=V \psi,
$$

where

$$
U=\lambda U_{0}, \quad V=\frac{4}{\Phi} \lambda^{3} U_{0}+\frac{2 i q_{x}}{\Phi^{3}} \lambda^{2} \sigma_{1}+\left(\frac{q_{x}}{\Phi^{3}}\right)_{x} \lambda V_{0},
$$


with

$$
U_{0}=\left(\begin{array}{cc}
-i & q \\
-q & i
\end{array}\right), \quad V_{0}=\left(\begin{array}{cc}
0 & -1 \\
1 & 0
\end{array}\right), \quad \sigma_{1}=\left(\begin{array}{ll}
0 & 1 \\
1 & 0
\end{array}\right), \Phi=\sqrt{1+q^{2}}
$$

\subsection{For $\lambda=\infty$}

Firstly, we define a matrix-value function $G(x, t)$ and a scalar function $p(x, t, \lambda)$ as

$G(x, t)=\sqrt{\frac{1+\Phi}{2 \Phi}}\left(\begin{array}{cc}1 & \frac{i(1-\Phi)}{q} \\ \frac{i(1-\Phi)}{q} & 1\end{array}\right), \quad p(x, t, \lambda)=x-\int_{x}^{\infty}(\Phi-1) \mathrm{d} x^{\prime}+4 \lambda^{2} t$

Similarly to [9], we introduce a transformation

$$
\psi(x, t, \lambda)=G(x, t) \mu(x, t, \lambda) \mathrm{e}^{-i \lambda p(x, t, \lambda) \sigma_{3}},
$$

where $\sigma_{3}=\left(\begin{array}{cc}1 & 0 \\ 0 & -1\end{array}\right)$. Then defining two Jost solutions $\mu_{j}(x, t, \lambda)(j=1,2)$ via

$$
\begin{aligned}
& \mu_{1}(x, t, \lambda)=\mathrm{I}+\int_{-\infty}^{x} \mathrm{e}^{-i \lambda[p(x, t, \lambda)-p(y, t, \lambda)] \hat{\sigma}_{3}} V_{1}(y, t, \lambda) \mu_{1}(y, t, \lambda) \mathrm{d} y, \\
& \mu_{2}(x, t, \lambda)=\mathrm{I}-\int_{x}^{\infty} \mathrm{e}^{-i \lambda[p(x, t, \lambda)-p(y, t, \lambda)] \hat{\sigma}_{3}} V_{1}(y, t, \lambda) \mu_{2}(y, t, \lambda) \mathrm{d} y,
\end{aligned}
$$

where $V_{1}=\frac{i q_{x}}{2 \Phi^{2}} \sigma_{1}$. Therefore, similarly to [9], we can obtain some properties of $\mu_{j}(x, t, \lambda)(j=1,2)$, which are useful in the following analysis, such as, the first column of $\mu_{1}(x, t, \lambda)$ and $\mu_{2}(x, t, \lambda)$ (denote by $\left[\mu_{1}\right]_{1}$ and $\left[\mu_{2}\right]_{1}$ ) is bounded and analytic in upper and lower half-plane (denote by $D_{1}$ and $D_{2}$ ) of $\lambda$, respectively; the symmetry condition

$\overline{\mu_{j}(x, t, \bar{\lambda})}=\sigma_{2} \mu_{j}(x, t, \lambda) \sigma_{2}, \mu_{j}(x, t,-\lambda)=\sigma_{1} \mu_{j}(x, t, \lambda) \sigma_{1}$; and the asymptotic behavior as $\lambda \rightarrow \infty, \mu_{j}=\mathrm{I}+\frac{1}{\lambda} D^{(1)}(x, t)+O\left(\frac{1}{\lambda^{2}}\right)$, where the off-diagonal entries of $D^{(1)}$ are $D_{12}^{(1)}=\frac{q_{x}}{4 \Phi^{3}}$ and $D_{21}^{(1)}=-\frac{q_{x}}{4 \Phi^{3}}$.

Next, we define the scattering matrix by

$$
\mu_{1}(x, t, \lambda)=\mu_{2}(x, t, \lambda) \mathrm{e}^{-i \lambda p(x, t, \lambda) \widehat{\sigma_{3}}} S(\lambda),
$$

here, $S(\lambda)=\left(\begin{array}{cc}a(\lambda) & -\overline{b(\bar{\lambda})} \\ b(\lambda) & \overline{a(\bar{\lambda})}\end{array}\right)$, with $\overline{a(\bar{\lambda})}=a(-\lambda)$ and $\overline{b(\bar{\lambda})}=-b(-\lambda)$.

Then, we obtain $a(\lambda)=\operatorname{det}\left(\left[\mu_{1}\right]_{1},\left[\mu_{2}\right]_{2}\right)$ (where $\operatorname{det}(A)$ means the determinate of a matrix A), and $a(\lambda)$ is analysis in $D_{1}$.

\subsection{For $\lambda=0$}

Introducing another transformation

$$
\psi(x, t, \lambda)=\mu^{0}(x, t, \lambda) \mathrm{e}^{-i \lambda\left(x+4 \lambda^{2} t\right) \sigma_{3}} .
$$


Similarly, we define two Jost solutions $\mu_{j}^{0}(x, t, \lambda)(j=1,2)$

$$
\begin{aligned}
& \mu_{1}^{0}(x, t, \lambda)=\mathrm{I}+\int_{-\infty}^{x} \mathrm{e}^{-i \lambda(x-y) \hat{\sigma}_{3}} V_{1}^{0}(y, t, \lambda) \mu_{1}^{0}(y, t, \lambda) \mathrm{d} y, \\
& \mu_{2}^{0}(x, t, \lambda)=\mathrm{I}-\int_{x}^{\infty} \mathrm{e}^{-i \lambda(x-y) \hat{\sigma}_{3}} V_{1}^{0}(y, t, \lambda) \mu_{2}^{0}(y, t, \lambda) \mathrm{d} y,
\end{aligned}
$$

where $V_{1}^{0}=-\lambda q V_{0}$. Then, $\left[\mu_{1}^{0}\right]_{1}$ and $\left[\mu_{2}^{0}\right]_{1}$ are bounded and analytic in $D_{1}$ and $D_{2}$, respectively. And as

$$
\lambda \rightarrow 0, \mu_{j}^{0}(x, t, \lambda)=\mathrm{I}+\left(\begin{array}{cc}
0 & -\int_{x}^{\infty} q \mathrm{~d} x^{\prime} \\
\int_{x}^{\infty} q \mathrm{~d} x^{\prime} & 0
\end{array}\right) \lambda+O\left(\lambda^{2}\right) .
$$

\subsection{The Relation between $\mu_{j}(x, t, \lambda)$ and $\mu_{j}^{0}(x, t, \lambda)$}

The function $\mu_{j}(x, t, \lambda)$ and $\mu_{j}^{0}(x, t, \lambda)$ related by

$$
\mu_{1}(x, t, \lambda)=G^{-1} \mu_{1}^{0}(x, t, \lambda) \mathrm{e}^{i \lambda c_{-} \sigma_{3}}, \quad \mu_{2}(x, t, \lambda)=G^{-1} \mu_{2}^{0}(x, t, \lambda) \mathrm{e}^{-i \lambda c_{+} \sigma_{3}},
$$

where $c_{-}=\int_{-\infty}^{x}(\Phi-1) \mathrm{d} x^{\prime}, c_{+}=\int_{x}^{+\infty}(\Phi-1) \mathrm{d} x^{\prime}$.

Then, we have $a(\lambda)=1+i \lambda c+O\left(\lambda^{2}\right), \lambda \rightarrow 0$, where

$c=c_{-}+c_{+}=\int_{-\infty}^{+\infty}(\Phi-1) \mathrm{d} x^{\prime}$ is a conserved quantity.

\subsection{The Riemann-Hilbert Problem in Variable $(y, t)$}

Defining

$$
M(x, t, \lambda)= \begin{cases}\left(\frac{\left[\mu_{1}\right]_{1}}{a(\lambda)},\left[\mu_{2}\right]_{2}\right), & \lambda \in D_{1}, \\ \left(\left[\mu_{2}\right]_{1}, \frac{\left[\mu_{1}\right]_{2}}{\overline{a(\bar{\lambda})}}\right), & \lambda \in D_{2} .\end{cases}
$$

As $\lambda \rightarrow \infty, \quad M(x, t, \lambda)=G^{-1}\left(\mathrm{I}+\left(\begin{array}{cc}-i c_{+} & -\int_{x}^{\infty} q \mathrm{~d} x^{\prime} \\ \int_{x}^{\infty} q \mathrm{~d} x^{\prime} & i c_{+}\end{array}\right) \lambda+O\left(\lambda^{2}\right)\right)$. Then in-

troducing the new scale $y(x, t)=x-\int_{x}^{\infty}(\Phi-1) \mathrm{d} x=x-c_{+}(x, t)$ and defining $\tilde{M}(y, t, \lambda)=M(x(y, t), t, \lambda)$.

We assume that the initial value $q_{0}(x)$ satisfies that it can make $a(\lambda)$ has the finite simple zeros $\lambda_{j}$. Therefore $\tilde{M}(y, t, \lambda)$ satisfies the following the Riemann-Hilbert problem:

- Analytic property: $\tilde{M}(y, t, \lambda)$ is analytic in $D_{1}$ and $D_{2}$, and continuous up to the boundary, $\lambda \in R$;

- Jump condition: $\tilde{M}_{+}(y, t, \lambda)=\tilde{M}_{-}(y, t, \lambda) \mathrm{e}^{-i \lambda\left(y+4 \lambda^{2} t\right) \widehat{\sigma_{3}}} J_{0}(\lambda)$, where

$$
J_{0}(\lambda)=\left(\begin{array}{cc}
1+|r(\lambda)|^{2} & \overline{r(\bar{\lambda})} \\
r(\lambda) & 1
\end{array}\right), \quad r(\lambda)=\frac{b(\lambda)}{a(\lambda)} ;
$$


- Normalization: $\tilde{M}(y, t, \lambda) \rightarrow \mathrm{I}, \lambda \rightarrow \infty$;

- Residue conditions: for some constant $C_{j}$,

$$
\operatorname{Res}_{\lambda=\lambda_{j}}[\tilde{M}]_{1}(y, t, \lambda)=C_{j} \mathrm{e}^{2 i \lambda_{j}\left(y+4 \lambda_{j}^{2} t\right)}[\tilde{M}]_{2}\left(y, t, \lambda_{j}\right) .
$$

Letting $\tilde{M}(y, t, \lambda)$ satisfies the above conditions, then this Riemann-Hilbert problem has a unique solution. Assume that the WKI equation satisfies the initial value conditions. We obtain the solution $q(x, t)$ for the initial value problem as follows:

$$
\begin{gathered}
-\int_{x(y, t)}^{\infty} q\left(x^{\prime}, t\right) \mathrm{d} x^{\prime}=\lim _{\lambda \rightarrow 0} \frac{\left(\tilde{M}(y, t, 0)^{-1} \tilde{M}(y, t, \lambda)\right)_{12}}{\lambda}, \\
x(y, t)=y-\lim _{\lambda \rightarrow 0} \frac{\left(\tilde{M}(y, t, 0)^{-1} \tilde{M}(y, t, \lambda)\right)_{11}-1}{i \lambda},
\end{gathered}
$$

then calculating derivative of Equation (14) with respect to $y$, we can obtain the solution $q(x(y, t), t)$.

\section{Cuspon Solutions}

\subsection{Algebraic System of N-Solitons}

From residue conditions (13), we have

$$
\left(\begin{array}{c}
\tilde{M}_{11}(y, t, \lambda) \\
\tilde{M}_{21}(y, t, \lambda)
\end{array}\right)=\left(\begin{array}{l}
1 \\
0
\end{array}\right)+\sum_{j=1}^{N} \frac{C_{j} \mathrm{e}^{2 i \lambda_{j}\left(y+4 \lambda_{j}^{2} t\right)}}{\lambda-\lambda_{j}}\left(\begin{array}{c}
\tilde{M}_{12}\left(y, t, \lambda_{j}\right) \\
\tilde{M}_{22}\left(y, t, \lambda_{j}\right)
\end{array}\right) .
$$

Combining the symmetry conditions and taking $\lambda=\bar{\lambda}_{j}$, then, by solving the algebraic system for $\tilde{M}_{12}\left(y, t, \lambda_{j}\right)$ and $\tilde{M}_{22}\left(y, t, \lambda_{j}\right)$, and recall the symmetry conditions, we can obtain $\tilde{M}(y, t, \lambda)$.

\subsection{One-Cuspon Solution}

When $N=1$, assuming $\lambda=i d(d>0)$, and letting $C_{1}=-2 d \mathrm{e}^{-2 y_{0}}$, where $d$ and $y_{0}$ are real constants. Denoting by $\delta=2 d\left(y-4 d^{2} t\right)+2 y_{0}$, we can obtain one-cuspon solution

$$
q(x(y, t), t)=\frac{2 \sinh (\delta)}{2-\cosh ^{2}(\delta)}, \quad x(y, t)=y-\frac{1}{d}(\tanh (\delta)-1) .
$$

For convenience to obverse solution figure, a numerical simulation example of such a one-cuspon solution $q(x, t)$ is given via Equation (17) in Figure 1.

\subsection{Two-Cuspon Solutions}

\subsubsection{Two-Cuspon Solution}

When $N=2$, assuming $\lambda_{1}=i a$ and $\lambda_{2}=i b(a>0, b>0)$, then letting $C_{1}=-a \mathrm{e}^{-2 \theta_{1}}$ and $C_{2}=-b \mathrm{e}^{-2 \theta_{2}}$, taking $\phi=2 a\left(y-4 a^{2} t\right)+2 \theta_{1}$ and $\psi=2 b\left(y-4 b^{2} t\right)+2 \theta_{2}$, here $a, b, \theta_{1}$ and $\theta_{2}$ are real constants. We obtain two-cuspon solution as follows: 


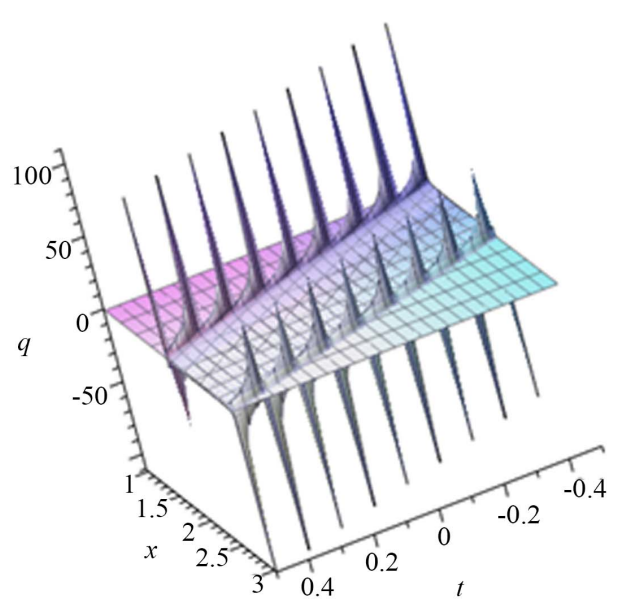

(a)

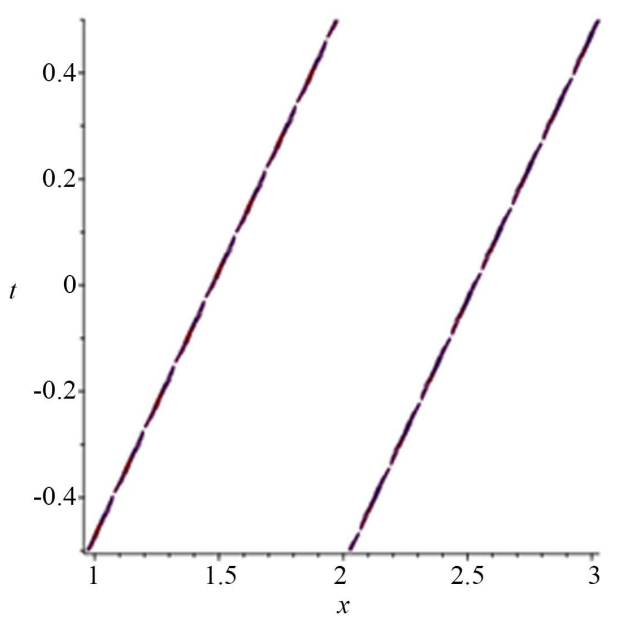

(b)

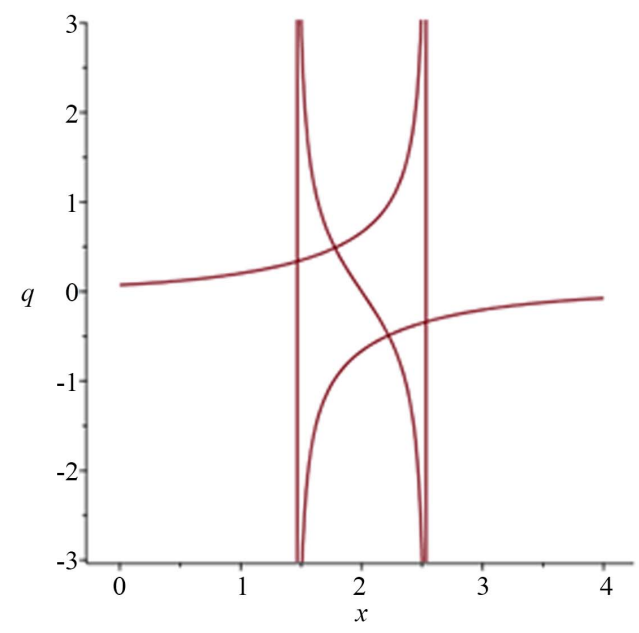

(c)

Figure 1. One-cuspon solution (17) with parameters $d=0.5$ and $y_{0}=0$. (a) The perspective view of the wave; (b) The corresponding contour pattern; (c) The pattern at $t=0$.

$$
\begin{aligned}
& q(x(y, t), t)=\frac{4\left(\frac{(a-b)^{2}}{4(a+b)^{2}} \mathrm{e}^{-\phi-\psi}-1\right)\left(\frac{\mathrm{e}^{-\phi}}{2}+\frac{\mathrm{e}^{-\psi}}{2}\right)\left[\left(\frac{(a-b)^{2}}{4(a+b)^{2}} \mathrm{e}^{-\phi-\psi}-1\right)^{2}-\left(\frac{\mathrm{e}^{-\phi}}{2}+\frac{\mathrm{e}^{-\psi}}{2}\right)^{2}\right]}{\left[\left(\frac{(a-b)^{2}}{4(a+b)^{2}} \mathrm{e}^{-\phi-\psi}-1\right)^{2}+\left(\frac{\mathrm{e}^{-\phi}}{2}+\frac{\mathrm{e}^{-\psi}}{2}\right)^{2}\right]^{2}-8\left(\frac{(a-b)^{2}}{4(a+b)^{2}} \mathrm{e}^{-\phi-\psi}-1\right)^{2}\left(\frac{\mathrm{e}^{-\phi}}{2}+\frac{\mathrm{e}^{-\psi}}{2}\right)^{2}}, \\
& x(y, t)=y+\frac{\frac{b \mathrm{e}^{-2 \phi}+a \mathrm{e}^{-2 \psi}}{2}+\frac{2 a b}{a+b} \mathrm{e}^{-\phi-\psi}+\frac{(a-b)^{4}}{8(a+b)^{3}} \mathrm{e}^{-2 \phi-2 \psi}}{a b\left[\left(\frac{(a-b)^{2}}{4(a+b)^{2}} \mathrm{e}^{-\phi-\psi}-1\right)^{2}+\left(\frac{\mathrm{e}^{-\phi}}{2}+\frac{\mathrm{e}^{-\psi}}{2}\right)^{2}\right]} .
\end{aligned}
$$

For convenience to obverse solution figure, a numerical simulation example of such a two-cuspon solution $q(x, t)$ is given via Equation (18) at Figure 2 . 

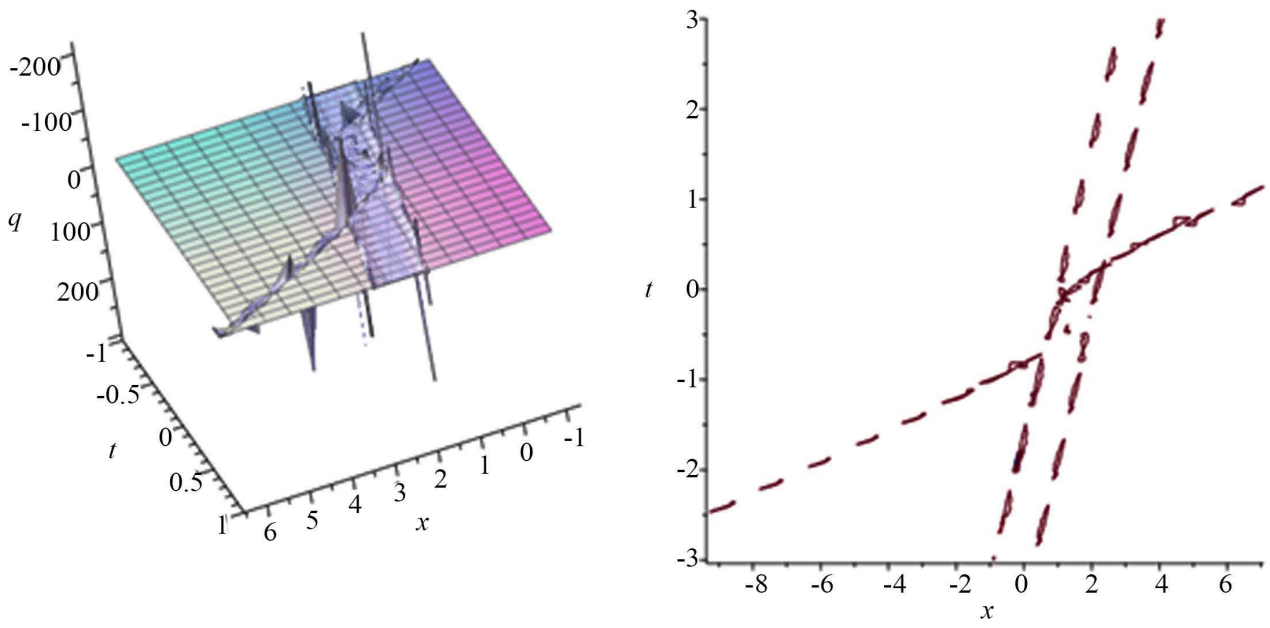

(a)

(b)

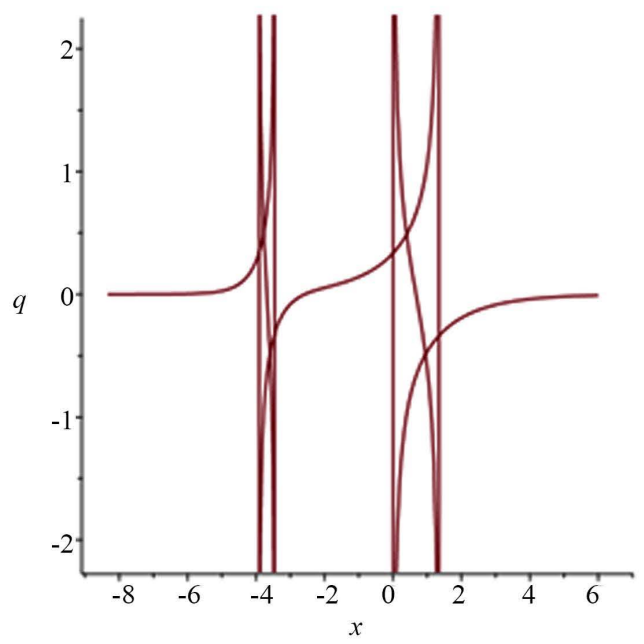

(c)

Figure 2. Two-cuspon solution (18) with parameters $a=0.4, b=1.2, \theta_{1}=0$ and $\theta_{2}=0$. (a) The perspective view of the wave; (b) The corresponding contour pattern; (c) The pattern at $t=-1.5$.

\subsubsection{Breather Solution}

Assuming that $\lambda_{1}=m+i n$ and $\lambda_{2}=-m+i n,(m>0, n>0)$, taking

$C_{1}=2(m+i n) \frac{n}{m} \mathrm{e}^{-2 \theta_{0}}, \phi_{1}=2 m\left(y+4\left(m^{2}-3 n^{2}\right) t\right)$ and

$\phi_{2}=2 n\left(y+4\left(3 m^{2}-n^{2}\right) t\right)+2 \theta_{0}$, here $m, n$ and $\theta_{0}$ are real constants, we can obtain the breather cuspon as follows:

$$
\begin{aligned}
& q(x(y, t), t)=\frac{4 m n \sin \left(\phi_{1}\right) \cosh \left(\phi_{2}\right)\left(n^{2} \sin ^{2}\left(\phi_{1}\right)-m^{2} \cosh ^{2}\left(\phi_{2}\right)\right)}{\left(n^{2} \sin ^{2}\left(\phi_{1}\right)+m^{2} \cosh ^{2}\left(\phi_{2}\right)\right)^{2}-8 m^{2} n^{2} \sin ^{2}\left(\phi_{1}\right) \cosh ^{2}\left(\phi_{2}\right)}, \\
& x(y, t)=y+\frac{m n}{m^{2}+n^{2}}\left(\frac{n \sin \left(2 \phi_{1}\right)-m \sinh \left(2 \phi_{2}\right)}{n^{2} \sin ^{2}\left(\phi_{1}\right)+m^{2} \cosh ^{2}\left(\phi_{2}\right)}+\frac{2}{m}\right) .
\end{aligned}
$$

For convenience to obverse solution figure, a numerical simulation example of such a breather solution $q(x, t)$ is given via Equation (19) at Figure 3. 


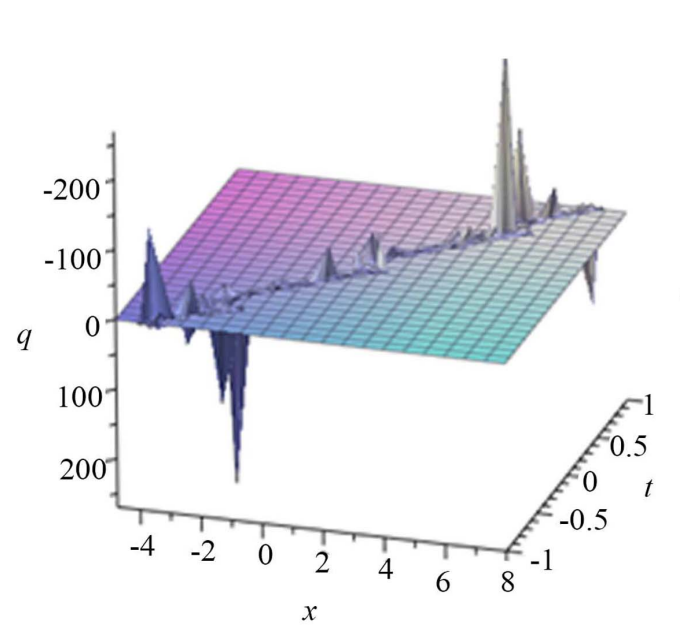

(a)

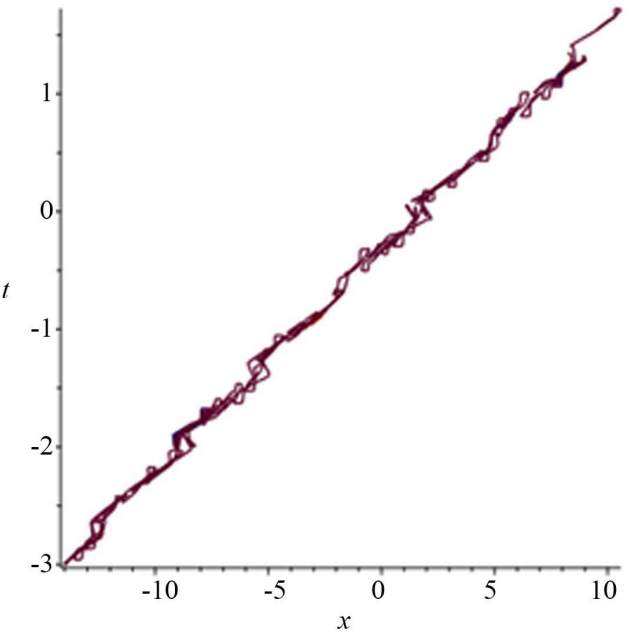

(b)

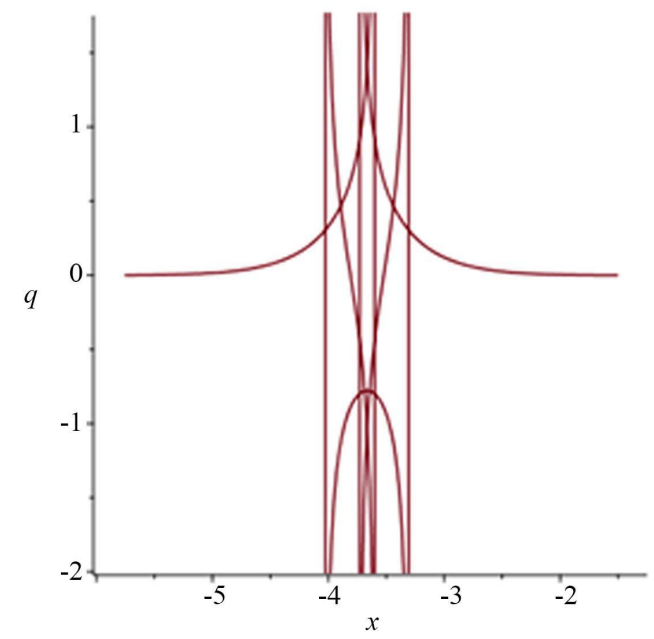

(c)

Figure 3. Breather solution (19) with parameters $m=0.2, n=1.2$ and $\theta_{0}=0$. (a) The perspective view of the wave; (b) The corresponding contour pattern; (c) The pattern at $t=-1$.

\subsection{Three-Cuspon Solution}

When $N=3$, assuming $\lambda_{1}=i a, \lambda_{2}=i b$ and $\lambda_{3}=i c(a>0, b>0, c>0)$, mean while taking $C_{1}=-a \mathrm{e}^{-2 \theta_{1}}, C_{2}=-b \mathrm{e}^{-2 \theta_{2}}, C_{3}=-c \mathrm{e}^{-2 \theta_{3}}$, and $\phi=-2 a\left(y-4 a^{2} t\right)+2 \theta_{1}, \quad \psi=-2 b\left(y-4 b^{2} t\right)+2 \theta_{2}, \quad \varphi=-2 c\left(y-4 c^{2} t\right)+2 \theta_{3}$, here $a, b, c, \theta_{1}, \theta_{2}$ and $\theta_{3}$ are real constants. The expression of three-soliton solution is very complicated, we don't write it in detail for space reason.

For convenience to obverse solution figure, a numerical simulation example of such a three-cuspon solution $q(x, t)$ is given in Figure 4 .

\section{Conclusion}

In this paper, the solutions of the WKI equation are recovered in terms of the solution of the matrix Riemann-Hilbert problem from the order $O(\lambda)$ at $\lambda \rightarrow 0$, like the case of the SP equation [9]. However, the novelty of our paper 


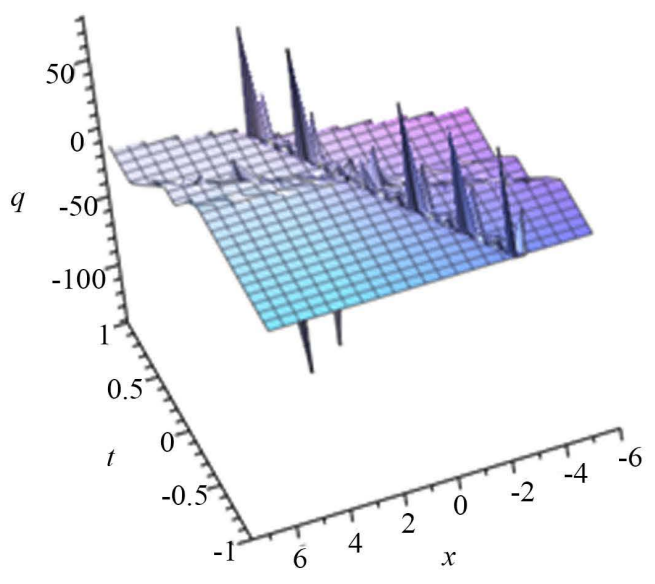

(a)

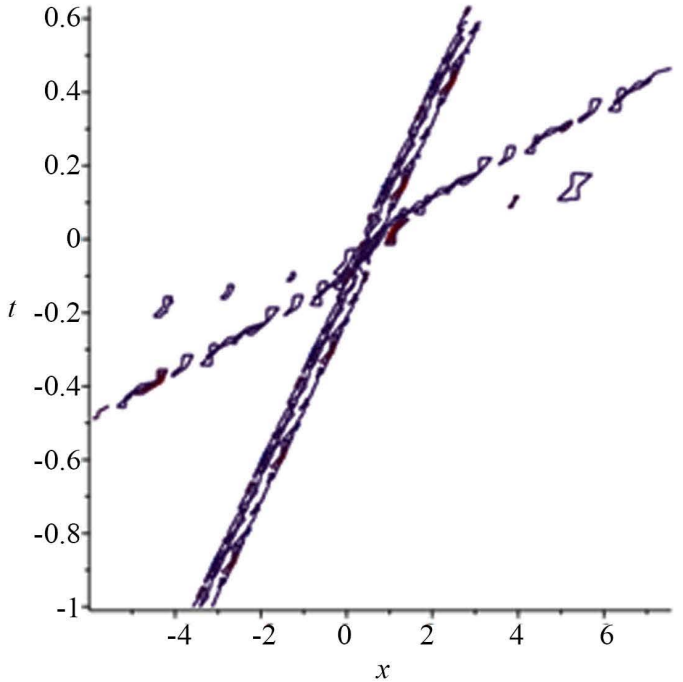

(b)

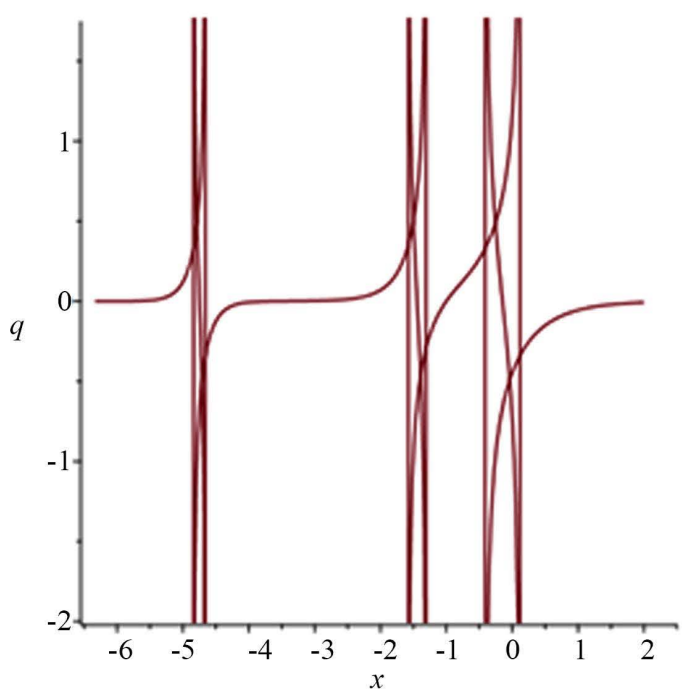

(c)

Figure 4. Three-cuspon solution with parameters $a=1, b=2, c=3, \theta_{1}=0, \theta_{2}=0$ and $\theta_{3}=0$. (a) The perspective view of the wave; (b) The corresponding contour pattern; (c) The pattern at $t=-0.2$.

is that $\lambda=0$ is non-singularity of the WKI equation. Then, one-cuspon solution, two types of two-cuspon solutions and three-cuspon solution are given via Riemann-Hilbert approach; one type of two-cuspon solutions is the breather, a novel solution of the WKI equation, which was not shown before. The numerical simulations of these solutions are given by choosing suitable parameters. Compared with the inverse scattering transform method, the calculation processes of the Riemann-Hilbert problem are more concise and efficient, and the most important advantage of the Riemann-Hilbert problem is analyzing the long-time asymptotic behavior of the solutions. The work of the analyzing long-time asymptotic behavior is beyond our aim in this paper, but we plan to complete this question in the future. 


\section{Conflicts of Interest}

The author declares no conflicts of interest regarding the publication of this paper.

\section{References}

[1] Wadati, M., Konno, K. and Ichikawa, Y. (1979) New Integrable Nonlinear Evolution Equations. Journal of the Physical Society of Japan, 47, 1689-1700. https://doi.org/10.1143/JPSJ.47.1698

[2] Wadati, M., Konno, K. and Ichikawa, Y. (1979) A Generalization of Inverse Scattering Method. Journal of the Physical Society of Japan, 46, 1965-1966. https://doi.org/10.1143/JPSJ.46.1965

[3] Ichikawa, Y., Konno, K. and Wadati, M. (1981) Nonlinear Transverse Oscillation of Elastic Beams under Tension. Journal of the Physical Society of Japan, 50, 1799-1802. https://doi.org/10.1143/JPSJ.50.1799

[4] Konno, K., Ichikawa, Y. and Wadati, M. (1981) A Loop Soliton Propagation along a Stretched Rope. Journal of the Physical Society of Japan, 50, 1025-1026. https://doi.org/10.1143/JPSJ.50.1025

[5] Qu, C.Z. and Zhang, D.B. (2005) The WKI Model of Type II Arises From Motion of Curves in $E^{3}$. Journal of the Physical Society of Japan, 74, 2941-2944. https://doi.org/10.1143/JPSJ.74.2941

[6] Debnath, L. (1984) Advances in Nonlinear Waves: The Loop Soliton. Konno, K and Jeffrey, A., Eds., Pitman Advanced Publishing Program.

[7] Zakharov, V.E. and Shabat, A. (1972) Exact Theory of Two-Dimensional Self-Focusing and One-Dimensional Self-Modulation of Waves in Nonlinear Media. Soviet Physics, 34, 12-69.

[8] Ichikawa, Y. (1982) A Relationship between the Ablowitz-Kaup-Newell-Segur and Wadati-Konno-Ichkawa Schemes of the Inverse Scattering Method. Journal of the Physical Society of Japan, 51, 3036-3041. https://doi.org/10.1143/JPSJ.51.3036

[9] Xu, J. (2018) Long-Time Asymptotics for the Short Pulse Equation. Journal of Differential Equations, 265, 3494-3532. https://doi.org/10.1016/j.jde.2018.05.009 